\title{
Coupling between phytoplankton growth and microzooplankton grazing in dilution experiments: potential artefacts
}

\author{
Andrés Gutiérrez-Rodríguez ${ }^{1}$, Mikel Latasa ${ }^{1,3, *}$, Baptiste Mourre $^{1}$, Edward A. Laws $^{2}$ \\ ${ }^{1}$ Institut de Ciències del Mar-ICM (CSIC), Passeig Marítim de la Barceloneta 37-49, 08003 Barcelona, Spain \\ ${ }^{2}$ Department of Environmental Sciences, School of the Coast and Environment, Louisiana State University, Baton Rouge, \\ Louisiana 70803, USA \\ ${ }^{3}$ Present address: Centro Oceanográfico de Gijón (IEO), Avda. Príncipe de Asturias 70 bis, 33213 Gijón, Spain
}

\begin{abstract}
Phytoplankton distribution is relatively constant in large areas of the surface ocean. In order to maintain this apparent stability, phytoplankton production and losses have to be balanced. Indeed, growth $\left(\mu_{0}\right)$ and grazing $(g)$ rates obtained simultaneously with the dilution technique are often tightly coupled. One problem with this approach is that growth and grazing are not independent in the ecological model on which the method is based (net growth rate $=\mu_{o}-g$ ). We evaluated to which extent this methodological artefact may influence the correlation between $\mu_{\mathrm{o}}$ and $g$ estimated using the dilution technique. Following a Monte-Carlo approach, we show that the methodological correlation can be substantial depending on: (1) the \% error in the measurement of the state variable $N_{\mathrm{D}}$ (e.g. chlorophyll a) and (2) the range $\left( \pm \mathrm{SD}\right.$ ) of the $\mu_{\mathrm{o}}$ and $g$ considered. As long as the error of $N_{\mathrm{D}}$ is small $(<10 \%)$, the measured correlation between growth and grazing closely reflects a true ecological relationship. For large errors, the dilution technique can yield a substantial correlation between both variables, regardless of their ecological relation. The influence of this methodological correlation decreases as the range of growth and grazing rate values increases. We developed a procedure to evaluate the ecological versus the methodological nature of the correlation observed between $\mu_{\mathrm{o}}$ and $g$. The application of this procedure to a data set obtained from a coastal site revealed that the high correlation observed $\left(\mathrm{r}_{\mathrm{S}}=0.881, \mathrm{p}<\right.$ 0.0001) reflected a true ecological relationship.
\end{abstract}

KEY WORDS: Dilution technique · Phytoplankton growth · Microzooplankton grazing · Coupling · Monte-Carlo simulation

Resale or republication not permitted without written consent of the publisher

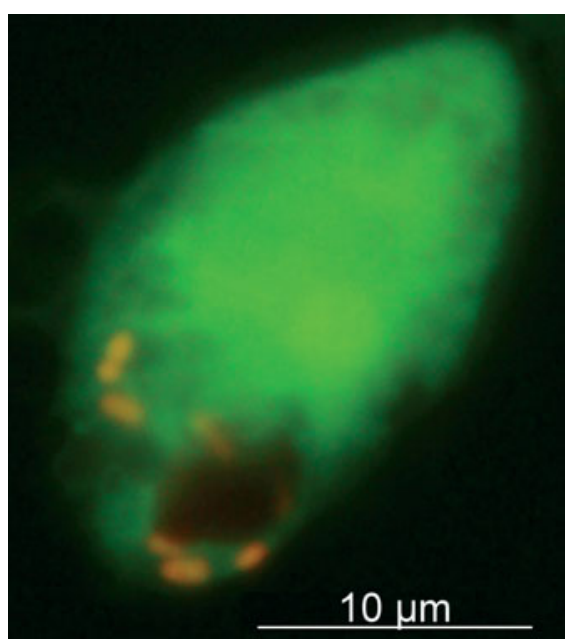

Epifluorescence microscope image of heterotrophic protist Oxyrrhis marina with ingested Synechococcus cells (orange).

Image: Jude Apple, Shannon Point Marine Centre

\section{INTRODUCTION}

Large areas of the ocean exhibit relatively constant phytoplankton biomass with little or no seasonal variation (Banse \& English 1994, Banse 2002). This observation requires phytoplankton gains and losses to be balanced. Among the different loss processes, microzooplankton grazing is considered to be the main factor controlling phytoplankton biomass (Calbet \& Landry 2004, Irigoien et al. 2005).

Different phytoplankton groups co-existing under the same environment display different growth rates 
(Furnas 1990, Strom \& Welschmeyer 1991, Latasa et al. 1997). This group-specific growth rate diversity implies that fast- and slow-growing taxa must be grazed accordingly to keep phytoplankton biomass constant. Parallel measurements of phytoplankton growth and grazing have supported this view by showing a good correlation between both variables, either for phytoplankton measured as a whole (McManus et al. 2007) or for major phytoplankton groups (Burkill et al. 1987, Strom \& Welschmeyer 1991, Latasa et al. 1997). In these studies, the experimental evidence was obtained with the dilution technique (Landry \& Hassett 1982), the only approach allowing the simultaneous estimation of phytoplankton growth and grazing rates.

The dilution technique is based on the serial dilution of natural seawater aiming to generate a gradient of grazing proportional to the dilution gradient established in each incubation bottle (Landry \& Hassett 1982, Landry et al. 1995). The apparent growth rate in each dilution treatment $\left(\mu_{D}\right)$, which is assumed to be exponential, is assessed from changes in a measured state variable of the phytoplankton population (chlorophyll a [chl a], carbon, cell counts, etc.) as:

$$
\mu_{\mathrm{D}}=\left(\frac{1}{t}\right) \ln \left(\frac{N_{\mathrm{D}(t)}}{N_{\mathrm{D}(0)}}\right)
$$

where $t$ is the incubation time and $N_{\mathrm{D}(0)}$ and $N_{\mathrm{D}(t)}$ are the chosen state variable of the phytoplankton population at the beginning and end of the incubation, respectively.

The apparent growth rate in each dilution treatment can be expressed as a function of the intrinsic growth rate $\left(\mu_{o}\right)$, fraction of unfiltered seawater or dilution gradient $\left(f_{\mathrm{D}}\right)$ and grazing $(g)$ following Model A:

$$
\mu_{\mathrm{D}}=\mu_{\mathrm{o}}-f_{\mathrm{D}} \times g
$$

Assuming that $g$ and $\mu_{0}$ are constant, a set of equations can be built to describe changes in $\mu_{\mathrm{D}}$ along the dilution gradient $\left(f_{\mathrm{D}}\right)$ (Landry \& Hassett 1982). Linear regression analysis allows the estimation of $\mu_{\mathrm{o}}$ and $g$, with their confidence limits, from $\mu_{\mathrm{D}}$ and the known $f_{\mathrm{D}}$. One problem with this approach is that phytoplankton $\mu_{\mathrm{o}}$ and $g$ are not mathematically independent, i.e. the errors in $\mu_{\mathrm{o}}$ and $g$ caused by an error in the calculation of $\mu_{\mathrm{D}}$ are not independent.

The goal of this work was to unveil the nature of the correlation between $\mu_{\mathrm{o}}$ and $g$ in data sets obtained from dilution experiments and to estimate the extent to which the calculated correlation reflects a methodological artefact rather than a true ecological link. We pursued this objective with 2 different types of approximation: (1) experimental field work and (2) a simulation exercise. In both cases, we modified either $\mu_{\mathrm{o}}$ or $g$ in order to uncouple their dynamics, yielding 2 independent, paired series of $\mu_{\mathrm{o}}$ and $g$. Our initial hypothesis stated that, when in situ ('true') $\mu_{\mathrm{o}}$ and $g$ are inde- pendent and follow independent dynamics, the correlation between $\mu_{0}$ and $g$ estimated with the dilution technique ('observed') will not be significantly different from zero. 'True' rates assume no error, and 'observed' rates include a measurement error. We also checked the variability of the methodological/artefactual correlation as a function of (1) the range of the measured $\mu_{o}$ and (2) the number of $\mu_{o}$ and $g$ pairs.

It is important to note that, in this study, we did not test the adequacy of the ecological model assumed in the dilution technique $\left(\mu_{\text {net }}=\mu_{\mathrm{o}}-g\right)$. Instead, we analyzed how the propagation of errors in the measurement of $\mu_{\text {net }}$ inherent to this accepted model, affects the correlation between $\mu_{\mathrm{o}}$ and $g$ reported in the field.

\section{MATERIALS AND METHODS}

Set up and calculations. For the experimental field work, we uncoupled $\mu_{\mathrm{o}}$ and $g$ by changing the irradiance of the dilution experiment incubations compared to the original conditions. We carried out 14 pairs of dilution experiments with surface seawater from Blanes Bay (NW Mediterranean). One of the pairs was incubated at saturating irradiance levels that simulated in situ conditions (high light, HL), and the other at limiting irradiance (low light, LL). We expected $\mu_{\mathrm{o}}$ to decrease under LL conditions (lower irradiance than in situ). Initially, we assumed grazing to be causally independent from phytoplankton growth rate and irradiance, although we are aware that microzooplankton grazing might respond to irradiance (Strom 2001).

For the simulation exercise, we generated noisecorrupted dilution experiments using the 14 estimates of $\mu_{\mathrm{o}}$ and $g$ obtained in the field. We then calculated $\mu_{\mathrm{o}}$ and $g$ in each simulated experiment ( $\mathrm{n}=14$ experiments $)$ and the Spearman correlation coefficient $\left(\mathrm{r}_{\mathrm{S}}\right)$ between $\mu_{\mathrm{o}}$ and $g$ in the parallel series. We repeated this simulation exercise following a Monte-Carlo approach in order to obtain the statistics for the $\mathrm{r}_{\mathrm{S}}$ (mean and SD). The correlation between 'true' $\mu_{\mathrm{o}}$ and $g$ was zero because grazing was kept constant, assuming the same level in all experiments. Thus, any correlation between 'observed' $\mu_{\mathrm{o}}$ and $g$ (hereafter the methodological $\mathrm{r}_{\mathrm{S}}$ correlation) would be due to the fact that errors in estimated $\mu_{\mathrm{o}}$ and $g$ are not independent. We calculated this methodological correlation for different errors in the measurement of the state variable (e.g. for $10 \%$ error, meaning that the measurement of chl $a$, the chosen state variable in our case, is made with an error of $10 \%$ ).

We also checked the variability of the methodological $r_{S}$ as a function of 2 factors: (1) the range of the measured $\mu_{0}$, i.e. how the range of the measured $\mu_{\mathrm{o}}$ in our dataset affects the methodological $r_{S}$ yielded by the simulation exercise; and (2) the number of $\mu_{\mathrm{o}}$ and $g$ 
pairs (n). This allowed us to interpret the nature (methodological versus ecological) of the correlation between $\mu_{0}$ and $g$, estimated with the dilution technique, assuming an error in the state variable measurement $\left(\% N_{\mathrm{D}}\right)$.

Field work. Monthly dilution experiments were performed during 2005 with surface seawater collected $1 \mathrm{~km}$ offshore in Blanes Bay (the Blanes Bay Microbial Observatory, $\mathrm{MO}, 41^{\circ} 40^{\prime} \mathrm{N}, 2^{\circ} 48^{\prime} \mathrm{E}$ ). Three additional experiments were carried out during the summer of 2007; one with surface seawater from the Blanes Bay $\mathrm{MO}$ and the other two with surface seawater retrieved $1 \mathrm{~km}$ offshore from Barcelona $\left(41^{\circ} 21^{\prime} \mathrm{N}, 2^{\circ} 10^{\prime} \mathrm{E}\right)$.

The experimental design followed that described in Landry et al. (1995) and Latasa et al. (2005) and included further modifications based on Gallegos (1989) 'three points' rationale. Briefly, the experiment included 5 bottles filled with appropriate quantities of filtered seawater to reach 90, 80, 70, 60 and $50 \%$ dilution. Three replicates of $100 \%$ unfiltered (whole) seawater along with the dilution series (Bottles 1 to 8 ) were nutrient amended with $\mathrm{f} / 2$ Guillard nutrient medium and ammonium to a final concentration of $6 \mu \mathrm{mol} \mathrm{l}^{-1}$. Urea and glucose were also added to a final concentration of $1.5 \mu \mathrm{mol} \mathrm{l} \mathrm{l}^{-1}$ and $1.0 \mu \mathrm{mol} \mathrm{l} \mathrm{l}^{-1}$, respectively. Three additional bottles of $100 \%$ whole seawater without nutrient amendment were included in the experimental design (Bottles 9 to 11).

Bottles were incubated for $24 \mathrm{~h}$ in a temperature and light controlled culture room in the laboratory. The diel light/dark cycle was adjusted to the length of the day on which the experiment was carried out. We prepared 2 complete dilution sets in parallel; one incubated under HL (320 $\mu \mathrm{mol}$ photon $\left.\mathrm{m}^{-2} \mathrm{~s}^{-1}\right)$, aiming to simulate in situ conditions, and a second set at LL conditions (10 to $15 \mu \mathrm{mol}$ photon $\mathrm{m}^{-2} \mathrm{~s}^{-1}$ ). We repeated this experiment on 15 occasions, covering most of the ecological conditions found at this coastal site throughout the year. One of the 15 experiments, conducted in December, did not work and was excluded from the analyses. We obtained 2 data sets of $14 \mu_{\mathrm{o}}$ and $g$ pairs, the first reflecting in situ $\mu_{\mathrm{o}}$ and $g$ and the second reflecting the rates obtained under LL conditions. The non-parametric spearman correlation coefficient $\left(\mathrm{r}_{\mathrm{S}}\right)$ between $\mu_{\mathrm{o}}$ and $g$ pairs was estimated for the HL and LL sets. We decided to use the non-parametric coefficient because of the low number of $\mu_{\mathrm{o}}$ and $g$ pairs available (14 pairs in our study).

Phytoplankton growth was assumed to be exponential and properly described by Model A (Eq. 2). Apparent growth rate of phytoplankton in each bottle was estimated following changes in pigment concentration, quantified using either High Pressure Liquid Chromatography (HPLC) or a Turner fluorometer. Sampling for pigment measurement, storage, extraction and quan- tification followed the protocol described in Latasa et al. (2005) and references therein.

Grazing was calculated as the slope of the Model A (Eq. 2) linear regression between $\mu_{\mathrm{D}}$ and $f_{\mathrm{D}}$ in the nutrient amended set of incubation bottles. When nonlinearities due to saturating feeding were detected, grazing was estimated as the difference between intrinsic growth rate $\left(\mu_{n}, y\right.$-axis intercept, phytoplankton growth rate in the nutrient amended bottles) and the mean $\mu_{\mathrm{D}}$ estimated from the non-diluted triplicate bottles $\left(\mu_{\text {net(n) }}\right)$ of the nutrient amended set. Intrinsic growth rate at ambient nutrient conditions $\left(\mu_{o}\right)$ was calculated as the mean $\mu_{\mathrm{D}}$ estimated from the non-diluted unamended triplicate $\left(\mu_{\text {net }}\right)$ plus the estimated grazing rate $\left(\mu_{o}=\mu_{\text {net }}+g\right)$. Grazing is assumed to be equal in nutrient amended and unamended bottles.

Monte-Carlo dilution experiments simulations. We first generated a set of 14 virtual dilution experiments applying Model A (Eq. 2), $\mu_{\mathrm{D}}=\mu_{\mathrm{o}}-f_{\mathrm{D}} \times g$, where $\mu_{\mathrm{o}}$ and $f_{\mathrm{D}}$ correspond to the 14 field experiments carried out at HL conditions and $g$ was assumed to be constant and calculated as the mean of the same 14 field experiments. Thus, these virtual dilution experiments were built under the premise of independence between 'true' $\mu_{\mathrm{o}}$ and $g$.

We then simulated dilution experiments giving errors to the state variable (chl a in our case). We considered a random error $\left(\varepsilon N_{\mathrm{D}}\right)$ associated with the state variable measurement in each bottle $\left(N_{\mathrm{D}}\right)$ and assumed this error to be normally distributed around a mean of zero and a standard deviation $(\sigma),\left(\varepsilon N_{\mathrm{D}} \sim\right.$ $\left.\mathrm{N}\left(0, \sigma^{2}\right)\right)$. The SD of the error was given by the \% error in the measurement of the state variable (i.e. the simulation of the $10 \%$ error means that our measurement of $N_{\mathrm{D}}$ is made with a $10 \%$ error). The condition that the noise-corrupted $N_{\mathrm{D}}$ measurement be positive further constrained the random error $\left(\varepsilon N_{D}\right)$. The $\mu_{D}$ in each simulated bottle incubated for $1 \mathrm{~d}$ was estimated following Eq. (3):

$$
\mu_{\mathrm{D}}=\ln \left(\frac{N_{\mathrm{D}(t)}}{N_{\mathrm{D}(0)}}\right)
$$

The error in the measurement of the state variable can be incorporated in this expression $\left(\varepsilon N_{\mathrm{D}(0)}\right.$ and $\varepsilon N_{\mathrm{D}(t))}$, error at the beginning and error at the end of each incubation). The error in $\mu_{D}\left(\varepsilon \mu_{D}\right)$ can be expressed as:

$$
\varepsilon \mu_{\mathrm{D}}=\ln \left[\frac{1+\varepsilon N_{\mathrm{D}(t)} / N_{\mathrm{D}(t)}}{1+\varepsilon N_{\mathrm{D}(0)} / N_{\mathrm{D}(0)}}\right]
$$

where $\frac{\varepsilon N_{\mathrm{D}(0)}}{N_{\mathrm{D}(0)}} \sim \mathrm{N}\left(0,0.1^{2}\right)$ and $\frac{\varepsilon N_{\mathrm{D}(t)}}{N_{\mathrm{D}(t)}} \sim \mathrm{N}\left(0,0.1^{2}\right)$ for $10 \%$ error in the measurement of the state variable (see Box 1 for details). 
Box 1. Formulation for a measurement error of $10 \%$

$$
\begin{aligned}
& \varepsilon N_{\mathrm{D}(t)} \sim \mathrm{N}\left\{0, \sigma^{2}=\left[0.1 \times N_{\mathrm{D}(t)}\right]^{2}\right\}=N_{\mathrm{D}(t)} \times \mathrm{N}\left[0, \sigma^{2}=(0.1)^{2}\right] \\
& \varepsilon N_{\mathrm{D}(0)} \sim \mathrm{N}\left\{0, \sigma^{2}=\left[0.1 \times N_{\mathrm{D}(0)}\right]^{2}\right\}=N_{0(t)} \times \mathrm{N}\left[0, \sigma^{2}=(0.1)^{2}\right]
\end{aligned}
$$

where $\mathrm{N}$ is a normal distribution with mean zero and variance $\sigma^{2}$. In our experimental protocol, $N_{0}$ in each bottle is calculated from $N_{0}$ in non-diluted bottles and the theoretical dilution factor as:

$N_{\mathrm{D}(0)}=N_{0} \times f_{\mathrm{D}}$

We estimated $\varepsilon \mu_{\mathrm{D}}$ as the difference between 'observed' $\mu_{\mathrm{D}}\left(\mu_{\mathrm{D} \_ \text {obs, }}\right.$ which includes the measurement error) and 'true' $\mu_{\mathrm{D}}\left(\mu_{\mathrm{D}_{\text {_truer }}}\right.$ without error $)$

$$
\begin{aligned}
& \mu_{\mathrm{D}}=\ln \left[\frac{N_{\mathrm{D}(t)}}{N_{\mathrm{D}(0)}}\right] \\
& \varepsilon \mu_{\mathrm{D}}=\ln \left[\frac{N_{\mathrm{D}(t)}+\varepsilon N_{\mathrm{D}(t)}}{N_{\mathrm{D}(0)}+\varepsilon N_{\mathrm{D}(0)}}\right]-\ln \left[\frac{N_{\mathrm{D}(t)}}{N_{\mathrm{D}(0)}}\right] \\
& \varepsilon \mu_{\mathrm{D}}=\ln \left[\frac{N_{D(t)}+N_{D(t)} \times \mathrm{N}\left(0,0.1^{2}\right)}{N_{\mathrm{D}(0)}+N_{\mathrm{D}(0)} \times \mathrm{N}\left(0,0.1^{2}\right)}\right]-\ln \left[\frac{N_{\mathrm{D}(t)}}{N_{\mathrm{D}(0)}}\right] \\
& \varepsilon \mu_{\mathrm{D}}=\ln \left[\frac{N_{\mathrm{D}(t)}\left[1+\mathrm{N}\left(0,0.1^{2}\right)\right]}{N_{\mathrm{D}(0)}\left[1+\mathrm{N}\left(0,0.1^{2}\right)\right]}\right]-\ln \left[\frac{N_{\mathrm{D}(t)}}{N_{\mathrm{D}(0)}}\right] \\
& \varepsilon \mu_{\mathrm{D}}=\ln \left[\frac{N_{\mathrm{D}(t)}}{N_{\mathrm{D}(0)}}\right]+\ln \left\{\frac{\left[1+\mathrm{N}\left(0,0.1^{2}\right)\right]}{\left[1+\mathrm{N}\left(0,0.1^{2}\right)\right]}\right\}-\ln \left[\frac{N_{\mathrm{D}(t)}}{N_{\mathrm{D}(0)}}\right] \\
& \varepsilon \mu_{\mathrm{D}}=\ln \left\{\frac{\left[1+\mathrm{N}\left(0,0.1^{2}\right)\right]}{\left[1+\mathrm{N}\left(0,0.1^{2}\right)\right]}\right\}
\end{aligned}
$$

We introduced this error term $\left(\varepsilon \mu_{D}\right)$ in Model A (Eq. 2) and obtained Model A1 for the nutrient amended set (Bottles 1 to 8 )

$$
\mu_{\mathrm{D}}=\mu_{\mathrm{n}}-f_{\mathrm{D}} \times g+\varepsilon \mu_{\mathrm{D}}
$$

and Model A2 for the unamended set (Bottles 9 to 11)

$$
\mu_{\mathrm{D}}=\mu_{\mathrm{o}}-f_{\mathrm{D}} \times g+\varepsilon \mu_{\mathrm{D}}
$$

where $\mu_{\mathrm{n}}$ and $\mu_{\mathrm{o}}$ are phytoplankton intrinsic growth rates in nutrient amended and unamended bottles, respectively.

At this stage, we had generated 14 virtual experiments with their corresponding $\mu_{\mathrm{D}}$ for each simulated incubation bottle (11 bottles in each of the 14 experiments), noise-corrupted with a random error in the state variable. We then estimated $\mu_{\mathrm{o}}$ and $g$ from each of these virtual experiments and calculated the $r_{S}$ correlation yielded by this set of 14 pairs of $\mu_{\mathrm{o}}$ and $g$. This $\mathrm{r}_{\mathrm{S}}$ is solely due to the fact that the errors in the estimation of $\mu_{\mathrm{o}}$ and $g$ caused by the error in the calculation of $\mu_{\mathrm{D}}$ are not independent; it does not reflect an ecological relation between $\mu_{\mathrm{o}}$ and $g$, but only the mathematical formulation of Model A assumed in the dilution method. The simulation exercise was repeated 1000 times to obtain the probability distribution of this methodological $r_{S}$ correlation for a specific error in the measurement of the state variable. We ran the same simulation for different errors in the measurement of $N_{\mathrm{D}(t)}$ and
$N_{\mathrm{D}(0)}(5$ to $60 \%$ ) and calculated the mean and SD of the methodological $r_{\mathrm{S}}$ for each error level.

Finally, we analyzed the variation of the methodological $r_{S}$ as a function of the number of $\mu_{o}$ values in the data set (n), the range of this series (measured as the SD) and a single parameter that combines the error in the measurement and the range of the $\mu_{\mathrm{o}}$ values as expressed in Eq. 7:

$$
\mathrm{L}=\frac{\% N_{\mathrm{D}}}{\mathrm{SD}}
$$

where $\% N_{\mathrm{D}}$ is the relative error in the measurement of the state variable and SD is the standard deviation of the $\mu_{\mathrm{o}}$ values.

\section{RESULTS AND DISCUSSION}

\section{Experimental results}

Growth and grazing rates results are shown in Table 1. Phytoplankton structure and associated seasonal dynamics are analyzed elsewhere (GutiérrezRodríguez 2008). Here, we will focus on the comparison between rates under in situ HL and LL conditions.

Growth rates were higher under in situ HL than under LL conditions (Wilcoxon rank test, $\mathrm{p}=0.0001$, Fig. 1A). As expected, this trend was not statistically significant for grazing (Wilcoxon rank test, $\mathrm{p}=0.194$, Fig. 1B). Nevertheless, grazing tended to be higher under HL than under LL conditions.

Growth and grazing rates from HL experiments were significantly correlated $\left(\mathrm{r}_{\mathrm{S}}=0.881, \mathrm{p}<0.0001, \mathrm{n}=14\right.$, Fig. 2A). Under LL conditions, this correlation was lower but statistically significant $\left(\mathrm{r}_{\mathrm{S}}=0.631, \mathrm{p}=0.016\right.$, $\mathrm{n}=14$, Fig. 2B).

\section{Simulation results}

We calculated the probability distribution of the $\mathrm{r}_{\mathrm{S}}$ between $\mu_{\mathrm{o}}$ and $g$ caused by the methodological approach, i.e. the methodological correlation. We obtained different probability distributions for the methodological $r_{S}$ (mean $\pm \mathrm{SD}$ ) depending on the magnitude of the measurement error of the state variable. This probability distribution for a $10 \%$ error in the measurement is shown in Fig. 3. The mean $\mathrm{r}_{\mathrm{S}}$ increases with the measurement error (Fig. 4). When the measurement error is low, the methodological mean $r_{S}$ between $\mu_{\mathrm{o}}$ and $g$ remains low (i.e. mean $\mathrm{r}_{\mathrm{S}}<0.25$ for an error of $10 \%$ ). For larger measurement errors, the methodological mean $r_{S}$ increases (i.e. error $>25 \%$; mean $r_{S}>0.43$ ). This trend is due to the propagation of the error in the estimation of $g$ that is caused by errors in the calcula- 
Table 1. Results of all field dilution experiments carried out in Blanes Bay and near Barcelona under in situ saturating (high light, $320 \mu \mathrm{mol}$ photon $\mathrm{m}^{-2}$ $\mathrm{s}^{-1}$ ) and limiting irradiances (low light, $10 \mu \mathrm{mol}$ photon $\mathrm{m}^{-2} \mathrm{~s}^{-1}$ ). Intrinsic growth rate under in situ $\left(\mu_{\mathrm{o}}\right)$ and nutrient amended conditions $\left(\mu_{\mathrm{n}}\right)$ and grazing $(g)$. Values are means \pm SEM. $p$-value and $\mathrm{r}^{2}$ from the Model A (Eq. 2) linear regression applied to estimate growth and grazing parameters

\begin{tabular}{|lccccc}
\hline $\begin{array}{l}\text { Day of } \\
\text { the year }\end{array}$ & $\mu_{\mathrm{o}}$ & $g$ & $\mu_{\mathrm{n}}$ & $\mathrm{r}^{2}$ & $\mathrm{p}$ \\
\hline \multicolumn{7}{l}{ High light } & & & & & \\
20 & $0.587 \pm 0.058$ & $0.154 \pm 0.040$ & $0.678 \pm 0.038$ & 0.492 & 0.024 \\
46 & $0.825 \pm 0.093$ & $0.204 \pm 0.072$ & $0.898 \pm 0.057$ & 0.507 & 0.001 \\
64 & $0.663 \pm 0.062$ & $0.036 \pm 0.04$ & $0.727 \pm 0.039$ & 0.052 & 0.254 \\
130 & $1.64 \pm 0.125$ & $1.40 \pm 0.125$ & $1.75 \pm 0.091$ & 0.945 & $<0.0001$ \\
158 & $0.760 \pm 0.117$ & $0.029 \pm 0.114$ & $0.519 \pm 0.106$ & 0.006 & 0.859 \\
$180^{\text {a }}$ & $1.40 \pm 0.049$ & $0.710 \pm 0.048$ & $1.425 \pm 0.036$ & 0.972 & 0.002 \\
$184^{\text {a }}$ & $0.905 \pm 0.07$ & $0.62 \pm 0.059$ & $1.14 \pm 0.055$ & 0.9306 & 0.0079 \\
187 & $1.91 \pm 0.055$ & $1.04 \pm 0.032$ & $1.93 \pm 0.027$ & 0.991 & $<0.0001$ \\
$191^{\text {a }}$ & $0.923 \pm 0.134$ & $0.485 \pm 0.120$ & $1.31 \pm 0.117$ & 0.968 & 0.016 \\
215 & $1.06 \pm 0.069$ & $0.572 \pm 0.048$ & $1.39 \pm 0.032$ & 0.959 & $<0.0001$ \\
256 & $1.16 \pm 0.109$ & $0.373 \pm 0.051$ & $1.57 \pm 0.049$ & 0.952 & $<0.0001$ \\
278 & $0.768 \pm 0.046$ & $0.354 \pm 0.022$ & $0.820 \pm 0.022$ & 0.947 & $<0.0001$ \\
309 & $0.651 \pm 0.058$ & $0.026 \pm 0.050$ & $0.737 \pm 0.041$ & 0.025 & 0.711 \\
339 & $0.808 \pm 0.107$ & $0.417 \pm 0.061$ & $0.843 \pm 0.059$ & 0.779 & 0.004 \\
Low light & & & & & \\
20 & $0.342 \pm 0.064$ & $0.321 \pm 0.063$ & $0.221 \pm 0.028$ & 0.80 & 0.003 \\
46 & $0.114 \pm 0.04$ & $0.100 \pm 0.017$ & $0.163 \pm 0.012$ & 0.84 & 0.048 \\
64 & $0.271 \pm 0.394$ & $0.032 \pm 0.280$ & $0.219 \pm 0.278$ & 0.06 & 0.370 \\
130 & $0.913 \pm 0.198$ & $0.901 \pm 0.195$ & $1.06 \pm 0.141$ & 0.76 & 0.005 \\
158 & $0.120 \pm 0.073$ & $0.148 \pm 0.068$ & $0.634 \pm 0.068$ & 0.21 & 0.248 \\
$180^{\text {a }}$ & $0.543 \pm 0.057$ & $0.276 \pm 0.035$ & $0.380 \pm 0.024$ & 1.00 & 0.001 \\
$184^{\text {a }}$ & $0.826 \pm 0.085$ & $0.423 \pm 0.082$ & $0.618 \pm 0.074$ & 0.81 & 0.039 \\
187 & $0.520 \pm 0.091$ & $0.352 \pm 0.056$ & $0.407 \pm 0.044$ & 0.87 & 0.002 \\
$191^{\text {a }}$ & $0.212 \pm 0.108$ & $0.484 \pm 0.075$ & $0.381 \pm 0.055$ & 0.86 & 0.001 \\
215 & $0.137 \pm 0.068$ & $0.119 \pm 0.056$ & $0.114 \pm 0.039$ & 0.40 & 0.093 \\
256 & $0.564 \pm 0.074$ & $0.276 \pm 0.045$ & $0.760 \pm 0.071$ & 0.69 & 0.075 \\
278 & $0.203 \pm 0.06$ & $0.456 \pm 0.060$ & $0.268 \pm 0.055$ & 0.96 & 0.023 \\
309 & $0.331 \pm 0.094$ & $0.228 \pm 0.094$ & $0.231 \pm 0.066$ & 0.51 & 0.071 \\
339 & $0.785 \pm 0.219$ & $0.613 \pm 0.213$ & $0.603 \pm 0.203$ & 0.80 & 0.04 \\
a Experiments carried out at the coast near Barcelona & & \\
& & & & & \\
\hline
\end{tabular}

tion of $\mu_{\mathrm{D}}$ being introduced into the estimated $\mu_{\mathrm{o}}$ with the same sign $\left(\mu_{\mathrm{o}}=\mu_{\text {net }}+g\right)$, i.e. an overestimation of $g$ will cause an overestimation of $\mu_{0}$. For small measurement errors, the error in $g$ is also small. Because $g$ is assumed to be constant for all experiments, this error is enough to randomize the rank of the $g$ vector but does not have the capability of modifying the rank of the $\mu_{\mathrm{o}}$ vector. Thus, for small measurement errors, the $\mu_{\mathrm{o}}$ vector conserves its rank free from the influence of the $g$ vector: the ranks remain independent.

As the magnitude of the measurement error increases, so does the error introduced in the estimated grazing. Then, the effect on $\mu_{o}$ is large enough to affect the rank of the $\mu_{\mathrm{o}}$ vector, and the correlation between the rank of $\mu_{o}$ and $g$ vectors increases. Thus, for large errors in the measurement of the state variable, the magnitude of the error in the 'observed' $g$ is large; the random rank of the $g$ vector influences the rank of $\mu_{\mathrm{o}}$ and yields a high $\mathrm{r}_{\mathrm{S}}$ correlation even with uncorrelated 'true' $\mu_{\mathrm{o}}$ and $g$. In summary, a high methodological $\mathrm{r}_{\mathrm{S}}$ is obtained when the measurement error leads to an error in the estimated grazing large enough to alter the rank of the $\mu_{\mathrm{o}}$ dataset.

This explanation suggests that the variability of the methodological $r_{S}$ should be sensitive not only to the magnitude of the measurement error (Fig. 4A), but also to the range (SD) of the values of $\mu_{0}$. Fig. 4B shows the different sensitivity of the methodological mean $r_{S}$ between three $\mu_{o}$
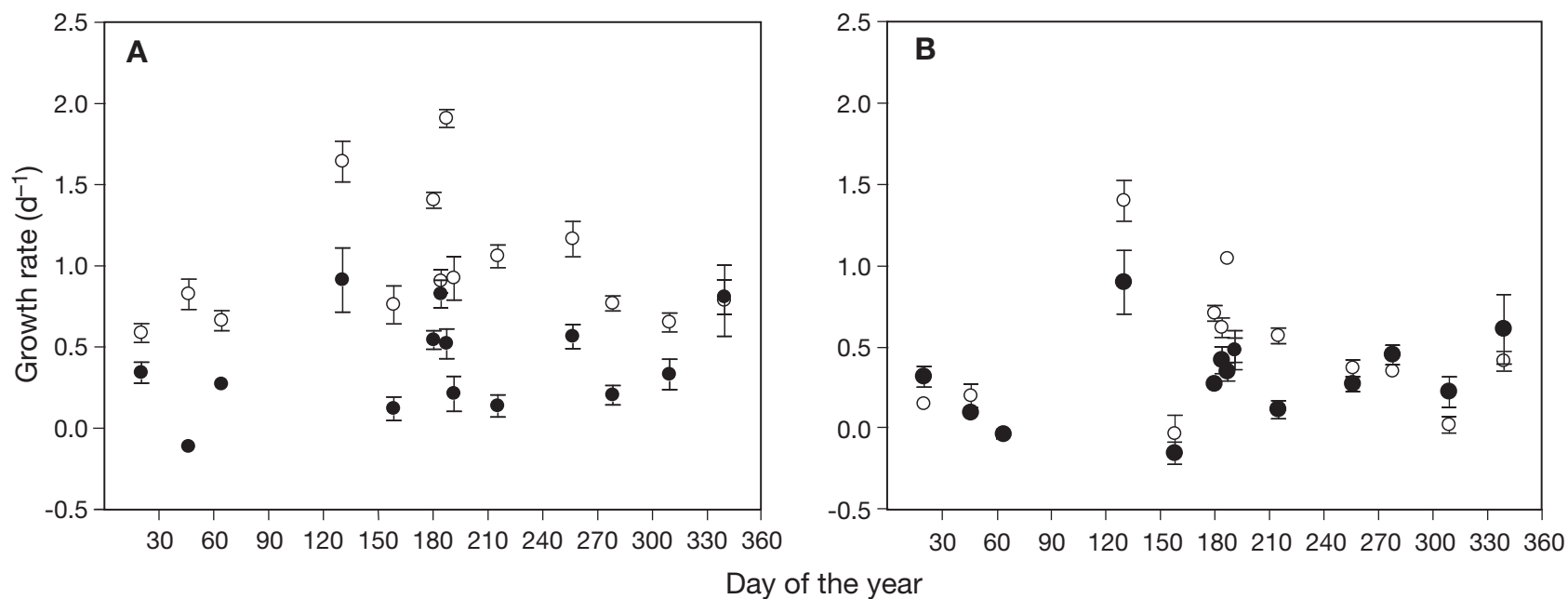

Fig. 1. (A) Growth and (B) grazing rate measured at (•) low light and (০) hight light incubation conditions at different times of the year. Error bars = SEM 

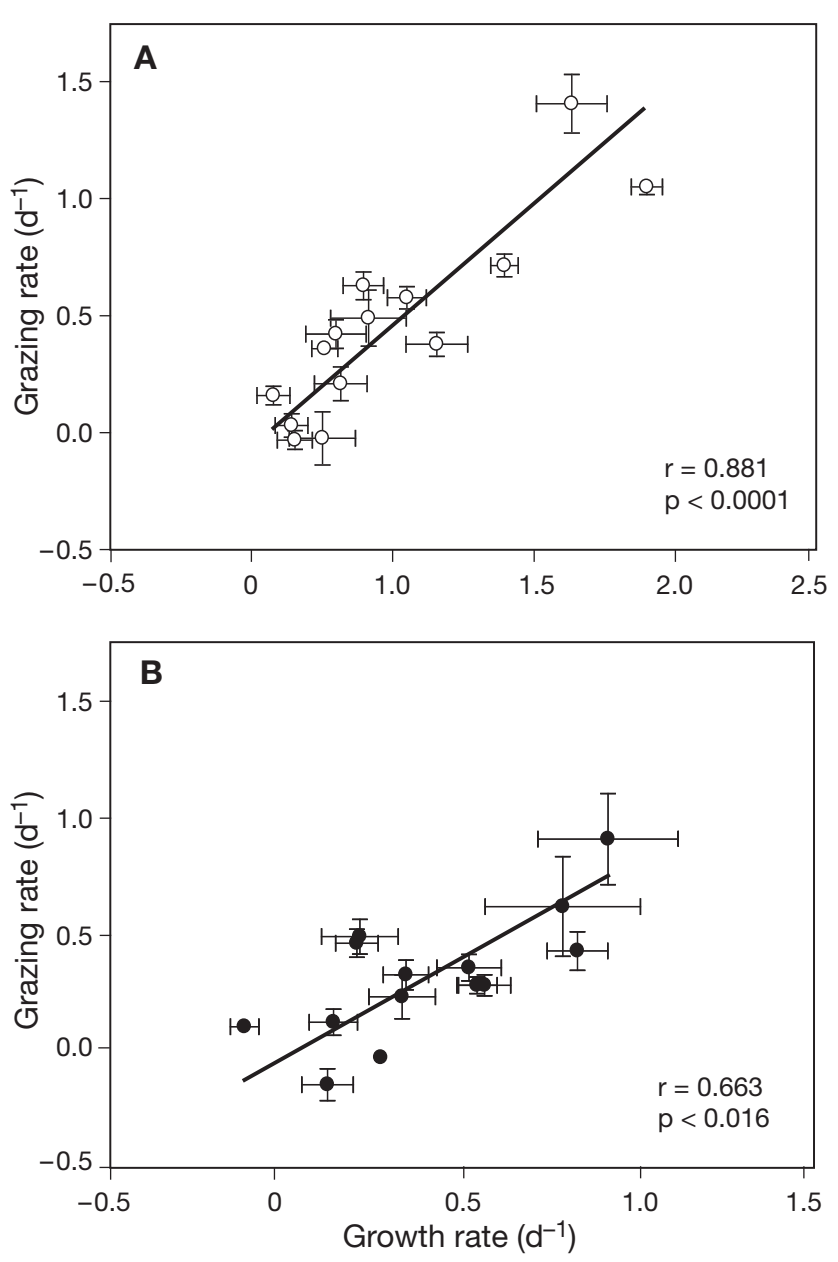

Fig. 2. The $r_{S}$ correlation between intrinsic growth and grazing rate obtained under (A) high light and (B) low light conditions. Error bars $=$ SEM

datasets with different range (SD). The higher the SD of $\mu_{o}$ values, the more 'robust' is its rank relative to the measurement error variability. Thus, when dealing with phytoplankton $\mu_{o}$ and $g$ obtained with the dilution technique, the correlation introduced by the method will be lower for data sets with higher variability (Fig. 4B). We identified 2 factors that determine to which extent the correlation observed between $\mu_{o}$ and $g$ is real or an artefact of the dilution technique: the error in the measurement of the state variable and the range (SD) of $\mu_{0}$.

We calculated a single parameter $\left(\mathrm{L}=\% N_{\mathrm{D}} / \mathrm{SD}\right)$ that summarizes the combined effect of these factors. Fig. 5 captures the r-methodological variability as a function of the parameter L. In our simulation exercise, $g$ is assumed to be constant and its variability $\left(V_{g}\right)$ is due to the error in the measurement of the state variable (the numerator in the parameter $\mathrm{L})$. The variability in $\mu_{\mathrm{o}}\left(V\left(\mu_{\mathrm{o}}\right)\right)$ accounts for the true variance of $\mu_{0}$ and the experimental error, and is quantified as the SD (the denominator

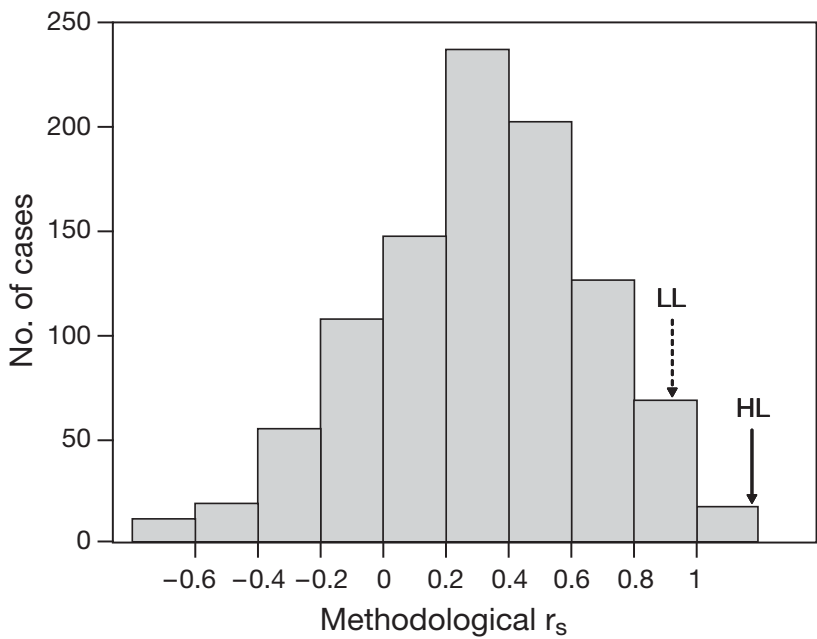

Fig. 3. Probability distribution of the $r_{S}$ between growth and grazing, assuming a relative error of $10 \%$ in the measurement of the state variable $(\mathrm{chl} \mathrm{a})$. Solid and dotted arrows indicate the $\mathrm{r}_{\mathrm{S}}$ calculated for our field experimental data set obtained from incubations under high light (HL) and low light (LL), respectively

of the parameter L). In summary, as long as $V_{g}$ remains low compared to $V_{\mathrm{x}}\left(\mu_{\mathrm{o}}\right)$, the r-methodological will remain low. This conclusion is implicit in the following expression (see Appendix 1 for derivation):

$$
V\left(\mu_{\mathrm{o}}\right)=V\left(\mu_{\mathrm{net}}\right)+V_{g}=\sigma^{2}\left(\mu_{\mathrm{o}}\right)+\frac{\sigma^{2}}{3}+\sigma^{2} / \Sigma(f-\bar{f})^{2}
$$

where $\sigma^{2}\left(\mu_{0}\right)$ is the true variance of $\mu_{0}, \sigma^{2}$ is the variance in $\mu_{\text {net }}$ caused by an experimental error, $\sigma^{2} / \Sigma(f-\bar{f})^{2}$ is the variance of $g, f$ is the dilution factor and $\bar{f}$ is the average of the dilution factors used in the dilution experiments. The methodological correlation between $\mu_{o}$ and $g$ will be small if $\sigma^{2}\left(\mu_{0}\right)$ is large compared to $\sigma^{2} / \Sigma(f-\bar{f})^{2}$.

The probability distribution of the methodological $r_{S}$ plotted in Fig. 3 shows that some $\mu_{o}$ and $g$ datasets might display a relatively high correlation even when the measurement error is low. This is also stressed by the $95 \%$ confidence intervals of the mean $\mathrm{r}_{\mathrm{S}}$ shown in Fig. 4A. The SD is linked to the number of dilution experiments (n) included in the simulation. The larger the $\mu_{\mathrm{o}}$ and $g$ vectors (higher $\mathrm{n}$ ), the more precise is the mean methodological $r_{S}$ yielded by the simulation. The mean $r$-methodological does not change with an increase in the number of $\mu_{o}$ and $g$ pairs, but the $95 \%$ confidence intervals of the mean become smaller (Fig. 5). This plot shows that the number of $\mu_{o}$ and $g$ pairs (n) influence the $95 \%$ confidence intervals of the mean methodological $\mathrm{r}_{\mathrm{S}}$ without affecting the mean itself.

Monte-Carlo simulation suggests that, in our field data set $\left(\mu_{o}=1.01 \pm 0.39 \mathrm{~d}^{-1}\right.$, mean $\left.\pm \mathrm{SD}, \mathrm{n}=14\right)$, the methodological correlation between $\mu_{o}$ and $g$ is low ( 0.23 for a $10 \%$ error in the measurement of the state 


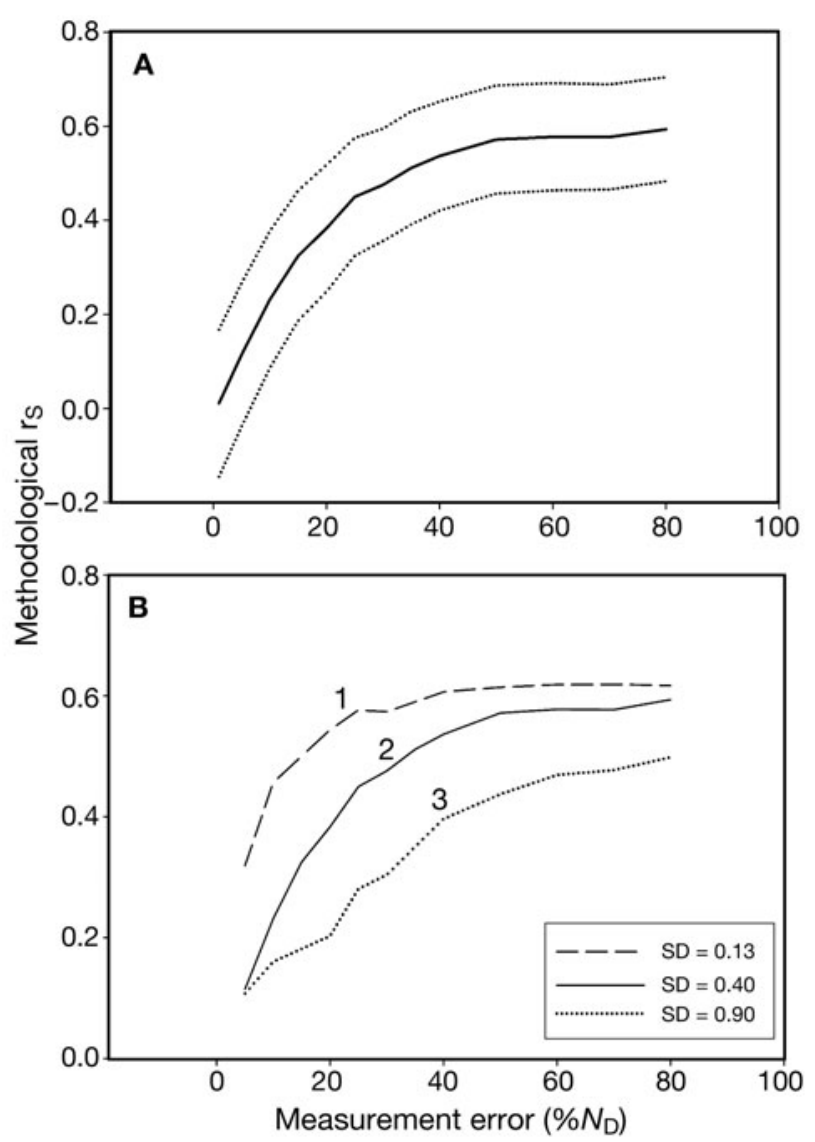

Fig. 4. Variability of the mean methodological correlation as a function of the \% error in the $N_{\mathrm{D}}$ measurements (based on 1000 simulations). The line is composed of discrete values distributed evenly every $5 \%$ error until $30 \% N_{\mathrm{D}}$ (i.e. 1,5 , $10 \ldots 30 \%$ ) and every $10 \%$ for errors $>30 \%$. (A) $\mu_{\mathrm{o}}$ and $g$ pairs estimated in field experiments under high light (HL) conditions ( $\mathrm{n}=14, \mathrm{SD}$ of $\left.\mu_{\mathrm{o}}=0.40\right)$. Dotted lines are the $95 \% \mathrm{CI}$ of the mean. (B) Effect of the ranges of $\mu_{\mathrm{o}}$ (expressed as SD) on the methodological $r_{S}$. Curve 2 (solid line, $\mathrm{n}=14, \mathrm{SD}$ of $\mu_{\mathrm{o}}=$ 0.40 ) is as in (A), Curve 1 (long dashed line, $\mathrm{n}=14, \mathrm{SD}$ of $\mu_{\mathrm{o}}=$ 0.13 ) and Curve 3 (dotted line, $\mathrm{n}=14, \mathrm{SD}$ of $\mu_{\mathrm{o}}=0.90$ ) are derived from $\mu_{0}$ and $g$ pairs estimated in field experiments under HL conditions

variable). The $\mathrm{r}_{\mathrm{S}}$ correlation between $\mu_{\mathrm{o}}$ and $g$ estimated at in situ HL conditions across the year in Blanes Bay was 0.881. The probability of obtaining such a high correlation with the dilution technique, if 'true' $\mu_{\mathrm{o}}$ and $g$ were independent, is $<0.1 \%$ assuming a reasonable measurement error of $10 \%$ (Latasa et al. 1996). Thus, the correlation we observed between $\mu_{o}$ and $g$ in Blanes Bay reflects a 'true' ecological relation between these variables. Under LL conditions, the $\mathrm{r}_{\mathrm{S}}$ between $\mu_{\mathrm{o}}$ and $g$ series estimated from parallel dilution experiments was 0.631 . The probability of obtaining an equal or higher correlation, assuming that in situ $\mu_{\mathrm{o}}$ and $g$ were uncorrelated, is $6.6 \%$ for a $10 \%$ measurement error. Our results show that the steady state relation between $\mu_{\mathrm{o}}$ and $g$ can be modified by changes in light conditions, at least on a daily basis. In our simulation, it was essential that $\mu_{o}$ and $g$ were independent. To assure independence, the $\mu_{\mathrm{o}}$ vector maintained the variability observed in Blanes Bay throughout the year, while the $g$ vector was assumed constant. However, the assumption of constant grazing might influence our results. We explored this possibility by running the same simulation exercise, but using randomly generated $\mu_{\mathrm{o}}$ and $g$ vectors, and we obtained the same results (Appendix 2).

\section{SUMMARY AND CONCLUSIONS}

We have demonstrated that the methodological errors in the estimation of $\mu_{o}$ and $g$ can affect the correlation between $\mu_{\mathrm{o}}$ and $g$ obtained with dilution experiments that is often reported in the field. We have shown that the extent to which this correlation is due to the methodological procedure of the dilution experiments or to a true ecological relationship depends on (1) the error in the measurement of the state variable and (2) the range of $\mu_{\mathrm{o}}$ and $g$ values included in the correlation. As long as measurement errors are small $(<10 \%)$, the correlation introduced by the methodological procedure remains small, with most of the uncertainty coming from the usually limited number of experiments. Under these conditions, the frequently observed correlation between both variables is reflecting a true ecological link. For large measurement errors, the dilution technique can yield a substantial correlation between obtained $\mu_{0}$ and $g$, regardless of their ecological relation. This methodological correlation is

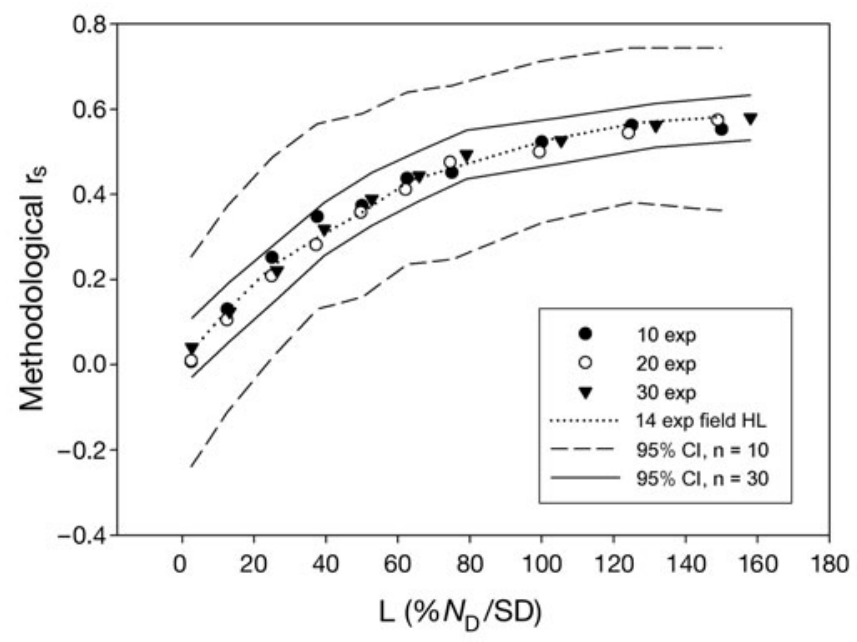

Fig. 5. Variability of the mean methodological $r_{S}$ as a function of the parameter $\mathrm{L}$ for different data sets. Selected data points are indicated by $(\boldsymbol{\nabla}) \mathrm{n}=30,(0) \mathrm{n}=20$ and $(\bullet) \mathrm{n}=10$ simulations. …... field data set obtained at high light (HL) conditions ( $\mathrm{n}=14$ experiments). The $95 \%$ CI for $\mathrm{n}=30$ (solid lines) and $n=10$ (dashed lines) are shown 
lower when the range of $g$ and $\mu_{\mathrm{o}}$ values is larger. In addition, we have shown that the number of $\mu_{\mathrm{o}}$ and $g$ pairs considered in the correlation analysis (n) determines the confidence interval of the mean methodological $r_{S}$ yielded by the simulation, without affecting the mean value. We have performed the same simulation exercise using a parametric correlation coefficient (Pearson) and reached the same conclusions.

Finally, we provide an easy and systematic way to assess the mean methodological correlation of a given set of $\mu_{o}$ and $g$ values from the range of $\mu_{\mathrm{o}}$ values (SD) and the measurement error in the state variable $\left(\% N_{\mathrm{D}}\right)$ assumed by the scientist. This analysis allows us to affirm with confidence that the correlation between $\mu_{o}$ and $g$ observed in Blanes Bay across the year reflects a true ecological relation. Moreover, this relation was affected by light. Irradiance affected $\mu_{\mathrm{o}}$ more strongly than $g$, allowing the former to escape from grazing control, at least on a daily basis.

Acknowledgements. This work was supported by the research project Eflubio (REN2002-04151-C02-01), funded by the Spanish Ministry of Education and Science. Financial support was provided by a PhD fellowship from the Spanish goverment to A.G.R. Different projects sustaining the Blanes Bay Microbial Observatory provided sampling facilities. We thank G. Vila for her help with flow cytometry analysis and V. Balague, C. Cardelús and I. Forn for their help during field sampling.

\section{LITERATURE CITED}

Banse K (2002) Should we continue to measure ${ }^{14} \mathrm{C}$-uptake by phytoplankton for another 50 years? Limnol Oceanogr Bull 11:45-46

Banse K, English DC (1994) Seasonality of coastal zone color scanner phytoplankton pigment in the offshore oceans. J Geophys Res 99(C4):7323-7345

Burkill PH, Mantoura RFC, Llewellyn CA, Owens NJD (1987)
Microzooplankton grazing and selectivity of phytoplankton in coastal waters. Mar Biol 93:581-590

Calbet A, Landry MR (2004) Phytoplankton growth, microzooplankton grazing, and carbon cycling in marine systems. Limnol Oceanogr 49(1):51-57

Furnas MJ (1990) In situ growth rates of marine phytoplankton: approaches to measurement, community and species growth rates. J Plankton Res 12:1117-1151

Gallegos CL (1989) Microzooplankton grazing on phytoplankton in the Rhode River, Maryland: nonlinear feeding kinetics. Mar Ecol Prog Ser 57:23-33

Gutiérrez-Rodríguez A (2008). Carbon fluxes through major phytoplankton groups in the sea. PhD dissertation, Universidad Politécnica de Catalunya, Barcelona

Irigoien X, Flynn KJ, Harris RP (2005) Phytoplankton blooms: a 'loophole' in microzooplankton grazing impact? J Plankton Res 27(4):313-321

> Landry MR, Hasset RP (1982) Estimating the grazing impact of marine microzooplankton. Mar Biol 67:283-288

> Landry MR, Kirshtein J, Constantinou J (1995) A refined dilution technique for measuring the community grazing impact of microzooplankton, with experimental tests in the central equatorial Pacific. Mar Ecol Prog Ser 120:53-63

Latasa M, Bidigare RR, Ondrusek ME, Kennicutt II MC (1996) HPLC annalysis on algal pigments: a comparison exercise among laboratories and recommendations for improved analytical performance. Mar Chem 51:315-324

Latasa M, Landry MR, Schluter L, Bidigare RR (1997) Pigment-specific growth and grazing rates of phytoplankton in the central equatorial Pacific. Limnol Oceanogr 42: 289-298

Latasa M, Moran X, Scharek R, Estrada M (2005) Estimating the carbon flux through main phytoplankton groups in the northwestern Mediterranean. Limnol Oceanogr 50: 1447-1458

> McManus GB, Costas BA, Dam HG, Lopes RM, Gaeta SA, Susini SM, Rosetta CH (2007) Microzooplankton grazing of phytoplankton in a tropical upwelling region. Hydrobiologia 575:69-81

Strom SL, Welschmeyer NA (1991) Pigment-specific rates of phytoplankton growth and microzooplankton grazing in the open subartic Pacific Ocean. Limnol Oceanogr 36(1): $50-63$

Strom SL (2001) Light-aided digestion, grazing and growth in herbivorous protists. Aquat Microb Ecol 23:253-261 
Appendix 1. Evaluation of the effect of variable $\mu_{\mathrm{o}}$ and $g$ initial conditions on the change of the methodological mean $\mathrm{r}_{\mathrm{S}}$ as a function of the measurement error

The basic Model A is

$$
\mu_{\mathrm{D}}=\mu_{\mathrm{o}}-f_{\mathrm{D}} \times g
$$

where $\mu_{\mathrm{o}}$ is the intrinsic growth rate of phytoplankton, $g$ is the grazing and $f_{\mathrm{D}}$ is the dilution factor (the fraction of undiluted seawater in each dilution bottle).

The parameter $\mu_{\text {net }}$ is the growth rate when $f=1$ (whole seawater without nutrient amendement). It is estimated from triplicate measurements. Hence

and

$$
\mu_{\text {net }}=\mu_{\mathrm{o}}-g
$$

$$
\mu_{\mathrm{o}}=\mu_{\text {net }}+g
$$

The value of $g$ is estimated from a linear regression of $\mu_{\text {net }}$ against $f$ in enriched seawater. The assumption is that grazing is not affected by the enrichment. The obvious concern is the built-in correlation between $\mu_{\mathrm{o}}$ and $g$ in Eq. (A3).

Since the estimation of $\mu_{\text {net }}$ is based on measurements that are independent of the measurements used to estimate $g$, it follows that

$$
V\left(\mu_{\mathrm{o}}\right)=V\left(\mu_{\text {net }}\right)+V_{g}
$$

where $V_{(x)}$ is the variance of $x$. In other words, the variance of the sum is the sum of the variances when the variables are independent.

In the virtual experiment we envision, $g$ is constant, and the variance of $\mu_{\text {net }}$ is calculated from

$$
V\left(\mu_{\text {net }}\right)=\sigma^{2}\left(\mu_{\mathrm{o}}\right)+\frac{\sigma^{2}}{3}
$$

where $\sigma^{2}$ is the variance of a growth rate measurement, and the factor of 3 accounts for the fact that the estimate is based on triplicate growth rate measurements.

The term $\sigma^{2}\left(\mu_{\mathrm{o}}\right)$ accounts for the true variance of $\mu_{\mathrm{o}}$, and $\sigma^{2} / 3$ accounts for experimental error. The variance of $g$ is given by the expression

$$
V_{g}=\frac{\sigma^{2}}{\sum(f-\bar{f})^{2}}
$$

where $\bar{f}$ is the average of the dilutions used in the dilution experiments. From Eq. (A4) it follows that

We conclude that

$$
V\left(\mu_{\mathrm{o}}\right)=\sigma^{2}\left(\mu_{\mathrm{o}}\right)+\frac{\sigma^{2}}{3}+\frac{\sigma^{2}}{\sum(f-\bar{f})^{2}}
$$

$$
\frac{V_{g}}{V\left(\mu_{\mathrm{o}}\right)}=\frac{\frac{\sigma^{2}}{\sum(f-\bar{f})^{2}}}{\sigma^{2}\left(\mu_{\mathrm{o}}\right)+\frac{\sigma^{2}}{3}+\frac{\sigma^{2}}{\sum(f-\bar{f})^{2}}}=\frac{1}{1+\frac{\sigma^{2}\left(\mu_{\mathrm{o}}\right)+\frac{\sigma^{2}}{3}}{\sigma^{2} / \sum(f-\bar{f})^{2}}} \text { (A8) }
$$

$V_{g}$ is less than $V\left(\mu_{\mathrm{o}}\right)$. However, if most of the variance of $\mu_{\mathrm{o}}$ is accounted for by the variance of $g$ (exclusively due to the random error in the measurement), then $\mu_{\mathrm{o}}$ and $g$ will be highly correlated. The ratio $V_{g} / V\left(\mu_{o}\right)$ is negatively correlated with $\sigma^{2}\left(\mu_{\mathrm{o}}\right)$ and positively correlated with $\sigma^{2}$. Thus, there will be little correlation between $g$ and $\mu_{\mathrm{o}}$ when $\sigma^{2}\left(\mu_{\mathrm{o}}\right)$ is large and/or $\sigma^{2}$ is small.

Appendix 2. Mathematical demonstration of the influence of the methodological error $\left(\% N_{\mathrm{D}}\right)$ on the correlation $\left(\mathrm{r}_{\mathrm{S}}\right)$ between $\mu_{\mathrm{o}}$ and $g$ when Model A is applied

We demonstrated that the range of $\mu_{o}$ vector affects the methodological $r_{S}$ (Fig. 5). However, the assumption of a constant grazing makes the rank of the $g$ vector very sensitive to the measurement error variability. In addition, natural variability of growth and grazing are of similar magnitude, while we have eliminated the $g$ variability in our approach. In order to evaluate the influence of the constant grazing initial conditions on our results, we performed the same simulation exercise, but using 2 independent vectors of $\mu_{o}$ and $g$ generated with a random variability. This variability was kept within the range observed for these variables in the field work. This simulation exercise showed that the pattern of change in the methodological mean $\mathrm{r}_{\mathrm{S}}$ as a function of the measurement error is independent of the variability of the $g$ vector (Fig. A1). This is because the influence of the measurement error variability on the rank of observed $\mu_{\mathrm{o}}$ and $g$, what we consider the methodological correlation, is lower when the variability of the true $\mu_{\mathrm{o}}$ and $g$ is higher.

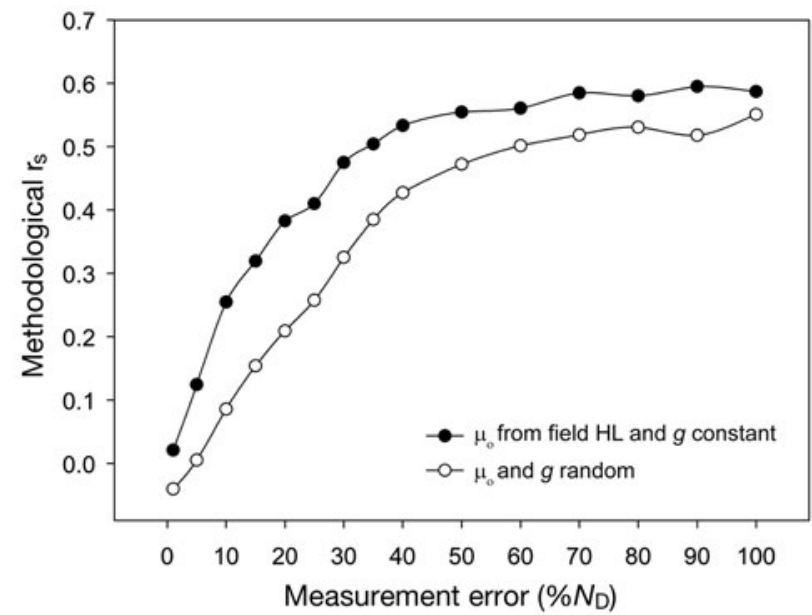

Fig. A1. Mean $r_{S}$ correlation coefficient between $\mu_{o}$ and $g$ produced by the methodological procedure as a function of the measurement error of the state variable. The 2 curves are associated with different initial $\mu_{\mathrm{o}}$ and $g$ vectors. $\bullet \mu_{\mathrm{o}}$ from field experiments with high light conditions and constant grazing; $\mathrm{O}$ : curve is obtained using 2 independent vectors $\left(\mu_{o}\right.$ and $g$ ) generated using a random variability with the range observed during field work 